\title{
On common extensions of valued fields
}

\author{
W. Mahboub \\ Lebanese International University \\ Mouseitbeh - PO Box: 146404 \\ Mazraa, Beirut, Lebanon.
}

\author{
A. Mansour \\ Lebanese International University \\ Mouseitbeh - PO Box: 146404 \\ Mazraa, Beirut, Lebanon.
}

\author{
M. Spivakovsky \\ Institut de Mathématiques de Toulouse \\ UMR 5219 du CNRS, \\ Université Paul Sabatier \\ 118, route de Narbonne \\ 31062 Toulouse cedex 9, France and \\ UMI CNRS 2001 LaSol, UNAM. \\ email: mark.spivakovsky@math.univ-toulouse.fr
}

August 7, 2020

\section{Introduction}

Let $(K, v)$ be a valued field, $\bar{K}$ an algebraic closure of $K$ and $\bar{v}$ an extension of $v$ to $\bar{K}$. Let $X$ be a transcendental element over $K$. With the aim of giving a characterization of residual transcendental extensions of $v$ to $K(X)$ [1, V. Alexandru and A. Zaharescu introduce the notion of "a minimal pair of definition". They prove that describing all such extensions is equivalent to describing all the minimal pairs $(a, \delta) \in \bar{K} \times \Gamma_{\bar{v}}$ (see Definition 2.5 below), where $\Gamma_{\bar{v}}$ is the value group of $\bar{v}$.

In [2], V. Alexandru, N. Popescu and A. Zaharescu investigate which pairs $(a, \delta) \in \bar{K} \times \Gamma_{\bar{v}}$ are minimal pairs. Given an extension $w$ of $K$ to $K(X)$, the authors define a common extension of $\bar{v}$ and $w$ to $\bar{K}(X)$, and they prove that there exists an integer, denoted by $[K: w]$, depending only on $v$ and $w$, and that the number of common extensions $\bar{w}$ is less than or equal to $[K: w]$ (see [2], Corollary 2.3 and its proof).

Another way of understanding the extensions $w$ of $v$ to $K(X)$ is via the theory of key polynomials introduced by S. Mac Lane (see [11] and [12]). The theory was introduced by S. Mac Lane in the case of discrete valuations of rank 1 and generalized by M. Vaquié to the case of arbitrary valuations. One important difference with the case of discrete rank 1 valuations is the presence of limit key polynomials. Another notion of key polynomials was introduced by F. H. Herrera, M. A. Olalla and M. Spivakovsky (see [7] or [8]). Yet another new notion of key polynomials was introduced in [5] and [15]. Comparison between these notions are given in [5] and [13. For more information about the key polynomial theory and its applications see [3], 4], [9], [14, [17] and [18]. 
It seems that the notions of key polynomials and minimal pairs are closely related. For recent studies on the relation between the two notions, see [16] and [23]. From Theorem 1.1 and Proposition 3.1 of [16], one can deduce that given a valuation $w$ which is residue-transcendental there exists a polynomial $Q$ such that $w=w_{Q}$ and every common extension $\bar{w}$ of $\bar{v}$ and $w$ can be described by a pair of definition $(a, \delta)$ where $a$ is a root of $Q$ and $\epsilon(Q)$ (see Section 4 for the definition of $w_{Q}$ and $\left.\epsilon(Q)\right)$. As well, one can deduce that such a $Q$ must be the last key polynomial in a complete sequence of key polynomials for $w$ (see Section 4 for the definition of a complete sequence of key polynomials).

In this paper we study the relation between key polynomials and minimal pairs. We also describe the classification given in Section 3 of [10] ("value-transcendental", "residuetranscendental", "valuation-transcendental" and "valuation-algebraic", see Definition 2.1 below) of all the possible extensions of $v$ from $K$ to $K(X)$ in terms of a complete sequence of key polynomials. Finally, we prove that the extension $w$ is residue-transcendental or value-transcendental if and only if the complete sequence of key polynomials for $w$ has a last element $Q$. In this case every common extension $\bar{w}$ of $\bar{v}$ and $w$ can be described by a pair of definition $(a, \delta)$ where $a$ is a root of $Q$. Moreover, we prove that in the case when the sequence of key polynomials does not admit a limit key polynomial, any root $a$ of $Q$ can be used to define a minimal pair $(a, \delta)$ that defines a common extension $\bar{w}$ of $\bar{v}$ and $w$.

In Section 2 we recall some basic facts about extensions of valuations. In Section 3 we give some properties relating common extensions and minimal pairs. Although the results of this section can also be found in [1] and 2], we reproduce them here in order to make the paper as self-contained as possible.

In Section 4 we recall some basic results on key polynomials. We use the construction of a complete set of key polynomials given in [15] and summarize briefly the main results of [15] used in the sequel.

In Section 5 we clarify the relation between the notion of key polynomials and the notion of minimal pair. We also describe the classification of the extension $w$ in terms of the complete sequence of key polynomials of $w$. This is accomplished in Corollaries 5.2 and 5.4 and Theorem 5.6. We also deduce that the number of common extensions $\bar{w}$ of $\bar{v}$ and $w$ is less than or equal to the number of roots of the last key polynomial of the sequence (see Corollary [5.7).

Finally, in Section [6 we study the relation between the roots of two consecutive key polynomials. We show that in the case when the sequence does not contain limit key polynomials, every root $a$ of the last key polynomial in the sequence can be used to construct a common extension $\bar{w}$ of $\bar{v}$ and $w$ (see Corollary 6.7).

\section{Basics and Notation}

Throughout this paper, we fix a valued field $(K, v)$, an algebraic closure $\bar{K}$ of $K$ and an extension $\bar{v}$ of $v$ to $\bar{K}$. We also fix a variable $X$ and an extension $w$ of $v$ to $K(X)$.

By Proposition 2.1 in [2], there exists a common extension of $\bar{v}$ and $w$ to $\bar{K}(X)$, that is, a valuation $\bar{w}$ on $\bar{K}(X)$ that is equal to $\bar{v}$ on $\bar{K}$ and to $w$ on $K(X)$. 
Let $\bar{w}$ be a common extension of $w$ and $\bar{v}$ to $\bar{K}(X)$.

We denote by $\Gamma_{v}, \Gamma_{\bar{v}}, \Gamma_{w}$ and $\Gamma_{\bar{w}}$ the respective value groups of $v, \bar{v}, w$ and $\bar{w}$. We have natural embeddings $\Gamma_{v} \subseteq \Gamma_{\bar{v}} \subseteq \Gamma_{\bar{w}}$ and $\Gamma_{v} \subseteq \Gamma_{w} \subseteq \Gamma_{\bar{w}}$.

We denote by $k_{v}, k_{\bar{v}}, k_{w}$ and $k_{\bar{w}}$ the respective residue fields of $v, \bar{v}, w$ and $\bar{w}$. We have natural extensions $k_{v} \subseteq k_{\bar{v}} \subseteq k_{\bar{w}}$, and $k_{v} \subseteq k_{w} \subseteq k_{\bar{w}}$.

Recall the following definitions from [10]:

Definition 2.1. The extension $w$ of $v$ to $K(X)$ is said to be valuation-algebraic if $\frac{\Gamma_{w}}{\Gamma_{v}}$ is a torsion group and $k_{w}$ is algebraic over $k_{v}$. The extension $w$ is said to be value-transcendental if $\frac{\Gamma_{w}}{\Gamma_{v}}$ has rational rank 1 and $k_{w}$ is algebraic over $k_{v}$. The extension $w$ is said to be residuetranscendental if $k_{w}$ has transcendence degree 1 over $k_{v}$ and $\frac{\Gamma_{w}}{\Gamma_{v}}$ is a torsion group. We will combine the value-transcendental case and the residue-transcendental case by saying that $w$ is valuation-transcendental if either $\frac{\Gamma_{w}}{\Gamma_{v}}$ has rational rank 1 or $k_{w}$ has transcendence degree 1 over $k_{v}$.

For a polynomial $f(X) \in K[X]$, we define the set $\mathcal{R}(f)$ by

$$
\mathcal{R}(f):=\{a \in \bar{K} / f(a)=0\} .
$$

For an element $y$ we denote by $y^{*}$ its image in the corresponding residue field.

We note that

$$
\Gamma_{\bar{v}}=\Gamma_{v} \bigotimes_{\mathbb{Z}} \mathbb{Q}
$$

and that $k_{\bar{v}}$ is an algebraic closure of $k_{v}$.

We define the set $M_{\bar{w}}:=\{\bar{w}(X-a) / a \in \bar{K}\}$.

Let $a \in \bar{K}$ and let $\delta$ be an element in an ordered group containing $\Gamma_{\bar{v}}$. We define the valuation $w_{(a, \delta)}$ in the following manner:

For a polynomial $f(X) \in \bar{K}[X]$, write the Taylor expansion of $f$ :

$$
f(X)=a_{n}(X-a)^{n}+\cdots+a_{1}(X-a)+a_{0} .
$$

Put $w_{(a, \delta)}(f(X))=\inf _{0 \leq j \leq n}\left\{\bar{v}\left(a_{j}\right)+j \delta\right\}$.

We define the set $S_{(a, \delta)}(f):=\left\{j \in\{1, \ldots, n\} / \bar{v}\left(a_{j}\right)+j \delta=w_{(a, \delta)}(f(X))\right\}$.

We note that if we fix $a \in \bar{K}$ and $\delta=\bar{w}(X-a)$, then for all $f \in \bar{K}[X]$ we have

$$
\bar{w}(f(X)) \geq w_{(a, \delta)}(f(X)) .
$$

If the inequality is strict then $\# S_{(a, \delta)}(f)>1$.

Remark 2.2. In the literature, minimal pairs are defined for residue transcendental valuations. In our case, we give the same definition but more generally for valuation-transcendental valuations.

Definition 2.3. For a valuation $\mu$ of $\bar{K}(X)$, we say that the pair $(a, \delta)$ is a pair of definition for the valuation $\mu$ if $\mu=w_{(a, \delta)}$. 
Lemma 2.4. Let $(a, \delta)$ be a pair of definition for a valuation $\mu$. Let $b \in \bar{K}$, and let $\delta^{\prime}$ be an element of an ordered group containing $\Gamma_{\bar{v}}$ as an ordered subgroup. Then $\left(b, \delta^{\prime}\right)$ is a pair of definition for $\mu$ if and only if $\delta^{\prime}=\delta$ and $\bar{v}(a-b) \geq \delta$.

Proof. Suppose first that $\delta^{\prime}=\delta$ and $\bar{v}(a-b) \geq \delta$. It is sufficient to prove that for all $c \in \bar{K}$, we have

$$
\mu(X-c)=\inf \{\delta, \bar{v}(b-c)\}
$$

Take an element $c \in \bar{K}$. We have

$$
\mu(X-c)=\inf \{\delta, \bar{v}(a-c)\} .
$$

Therefore we have to prove that

$$
\inf \{\delta, \bar{v}(b-c)\}=\inf \{\delta, \bar{v}(a-c)\} .
$$

We will use the equality $a-c=a-b+b-c$ and the fact that $\bar{v}(a-b) \geq \delta$.

If $\delta>\bar{v}(b-c)$ then $\bar{v}(a-b)>\bar{v}(b-c)=\bar{v}(a-c)$, and (2.3) is proved.

Otherwise, if $\delta \leq \bar{v}(b-c)$ then $\bar{v}(a-c) \geq \inf \{\bar{v}(a-b), \bar{v}(a-c)\} \geq \delta$, and again (2.3) is proved.

Conversely, suppose that $\left(b, \delta^{\prime}\right)$ is a pair of definition for $\mu$. We have:

$$
\delta=\mu(X-a)=w_{\left(b, \delta^{\prime}\right)}(X-a)=\inf \left\{\delta^{\prime}, \bar{v}(a-b)\right\},
$$

hence $\delta \leq \bar{v}(a-b)$ (this proves the second statement), and $\delta \leq \delta^{\prime}$. On the other hand, we have

$$
\delta^{\prime}=\mu(X-b)=w_{(a, \delta)}(X-b)=\inf \{\delta, \bar{v}(a-b)\},
$$

hence $\delta^{\prime} \leq \delta$ and we get the desired equality.

Let $\mu=w_{(a, \delta)}$ for a certain pair $(a, \delta)$ as above. We define the degree of $\mu$

$$
\mathcal{D}(\mu):=\min \left\{[K(b): K] / b \in \bar{K}, \mu=w_{(b, \delta)}\right\} .
$$

Definition 2.5. A pair of definition $(a, \delta)$ for $\mu$ is said to be minimal if $[K(a): K]=\mathcal{D}(\mu)$. We also say in this case that $(a, \delta)$ is a minimal pair of definition for $\mu$.

\section{Minimal Pairs}

In this section, we give some properties of common extensions and minimal pairs. Keep the notation of the previous section.

The following result is in [1], Proposition 1.1.

Lemma 3.1. Suppose that $\Gamma_{\bar{v}}=\Gamma_{\bar{w}}$ and let $a \in \bar{K}$. The following conditions are equivalent:

(a) $\bar{w}(X-a)=\operatorname{Max}\left(M_{\bar{w}}\right)$.

(b) for each $b \in \bar{K}$ with $\bar{w}(X-a)=\bar{v}(b)$, the element $\left(\frac{X-a}{b}\right)^{*}$ is transcendental over $k_{\bar{v}}$. 
Proof. Suppose (a) is satisfied. Let $b \in \bar{K}$ be such that $\bar{w}(X-a)=\bar{v}(b)$. Put $t=\left(\frac{X-a}{b}\right)^{*}$. If $t$ is algebraic over $k_{\bar{v}}$ then $t \in k_{\bar{v}}$, since $k_{\bar{v}}$ is algebraically closed. Choose $c \in \bar{K}$ so that $c^{*}=t$. Now we have $\bar{w}\left(\frac{X-a}{b}-c\right)>0$, that is, $\bar{w}(X-a-c b)>\bar{w}(X-a)$, which is a contradiction.

Suppose (b) is satisfied and suppose that there exists $c \in \bar{K}$ such that $\bar{w}(X-c)>\bar{w}(X-a)$. Then $\bar{w}(X-a+a-c)>\bar{w}(X-a)=\bar{v}(a-c)$. Therefore we have $\bar{w}\left(\frac{X-a}{c-a}-1\right)>0$ and hence $\left(\frac{X-a}{c-a}\right)^{*}=1$ in $K_{\bar{v}}$, which is a contradiction.

Lemma 3.2. Suppose that $\Gamma_{\bar{v}} \varsubsetneqq \Gamma_{\bar{w}}$. Then $\operatorname{Max}\left(M_{\bar{w}}\right)$ exists and $\operatorname{Max}\left(M_{\bar{w}}\right) \notin \Gamma_{\bar{v}}$. Moreover, $\operatorname{Max}\left(M_{\bar{w}}\right)$ is the unique element in $M_{\bar{w}}$ that does not belong to $\Gamma_{\bar{v}}$.

Proof. Let $f \in \bar{K}[X]$ be such that $\bar{w}(f(X)) \notin \Gamma_{\bar{v}}$. Write $f(X)=c \prod_{i=1}^{n}\left(X-c_{i}\right)$. There exists $i$, $1 \leq i \leq n$, such that $\bar{w}\left(X-c_{i}\right) \notin \Gamma_{\bar{v}}$.

Let $a=c_{i}$ for such an $i$ and let $\delta=\bar{w}(X-a)$.

Let $b \in \bar{K}$. If $\bar{w}(X-b)>\delta$. We can write $\bar{w}(X-a+a-b)>\delta$. Then $\bar{v}(a-b)$ is equal to $\delta$ which is impossible.

$$
\text { If } \bar{w}(X-b)<\delta \text {, then } \bar{w}(X-b)=\bar{v}(a-b) \in \Gamma_{\bar{v}} \text {. }
$$

It is easy to see that $w$ is a residue-transcendental extension of $v$ if and only if $\bar{w}$ is a residue-transcendental extension of $\bar{v}$. Similarly, $w$ is a value-transcendental extension of $v$ if and only if $\bar{w}$ is a value-transcendental extension of $\bar{v}$.

Using this fact together with Lemma 3.1 and Lemma 3.2 we deduce

Proposition 3.3. (a) $w$ is residue-transcendental extension of $v$ if and only if $\Gamma_{\bar{v}}=\Gamma_{\bar{w}}$ and $M_{\bar{w}}$ has a maximal element.

(b) $w$ is value-transcendental extension of $v$ if and only if $\Gamma_{\bar{v}} \varsubsetneqq \Gamma_{\bar{w}}$. In this case, again, $M_{\bar{w}}$ has a maximal element.

(c) $w$ is a valuation-algebraic extension of $v$ if and only if $M_{\bar{w}}$ does not have a maximal element.

Proposition 3.4. The set $M_{\bar{w}}$ has a maximal element $\delta$ if and only if $\bar{w}=w_{(a, \delta)}$ for some $(a, \delta) \in \bar{K} \times \Gamma_{\bar{w}}$ with $\bar{w}(X-a)=\delta$.

Proof. Suppose that $M_{\bar{w}}$ has a maximal element $\delta$.

Suppose first that $\delta \notin \Gamma_{\bar{v}}$. Take an $f(X) \in \bar{K}[X]$. Write the Taylor expansion

$$
f(X)=a_{n}(X-a)^{n}+\cdots+a_{0} .
$$

For $0 \leq \ell_{1}<\ell_{2} \leq n$ we must have $\bar{v}\left(a_{\ell_{1}}\right)+\ell_{1} \delta \neq \bar{v}\left(a_{\ell_{2}}\right)+\ell_{2} \delta$. Hence $\bar{w}(f(X))=w_{(a, \delta)}(f(X))$. 
Now suppose that $\delta \in \Gamma_{\bar{v}}$. Then we are in the case when $w$ is a residue-transcendental extension of $v$. We always have the inequality (2.2), so we only need to rule out the strict inequality in (2.2). Suppose there exists a polynomial $f(X) \in \bar{K}[X]$, such that

$$
\bar{w}(f(X))>w_{(a, \delta)}(f(X)) .
$$

Choose a monic polynomial $f$ of minimal degree satisfying the strict inequality (3.1). Write the Taylor expansion $f(X)=a_{n}(X-a)^{n}+a_{n-1}(X-a)^{n-1}+\cdots+a_{0}$ with $a_{n}=1$.

$$
\begin{aligned}
& \text { Write } f=\sum_{j \in S_{(a, \delta)}(f)} a_{j}(X-a)^{j}+\sum_{j \notin S_{(a, \delta)}(f)} a_{j}(X-a)^{j} \text {. We have } \\
& \qquad \begin{aligned}
\bar{w}\left(\sum_{j \in S_{(a, \delta)}(f)} a_{j}(X-a)^{j}\right) & \geq \inf \left\{\bar{w}(f), \bar{w}\left(\sum_{j \notin S_{(a, \delta)}(f)} a_{j}(X-a)^{j}\right)\right\} \\
& >w_{(a, \delta)}(f)=w_{(a, \delta)}\left(\sum_{j \in S_{(a, \delta)}(f)} a_{j}(X-a)^{j}\right) .
\end{aligned}
\end{aligned}
$$

Thus replacing $f(X)$ by $\sum_{j \in S_{(a, \delta)}(f)} a_{j}(X-a)^{j}$ does not affect the strict inequality (3.1). Hence we may assume that for all $j, 0 \leq j \leq n$, if $a_{j} \neq 0$ then $j \in S_{(a, \delta)}(f)$. We will make this assumption from now on. In particular, we have $w_{(a, \delta)}(f)=n \delta$.

Now by Lemma 3.1 there exists $b$ such that $t=\left(\frac{X-a}{b}\right)^{*}$ is transcendental over $k_{\bar{v}}$.

Using the fact that $\delta=\bar{w}(X-a)=\bar{v}(b)$, we see that $w_{(a, \delta)}\left(\frac{f}{b^{n}}\right)=n \delta-n \delta=0$. Hence for each $j \in\{0, \ldots, n\}$ we have $\bar{w}\left(\frac{a_{j}}{b^{n-j}} \frac{(X-a)^{j}}{b^{j}}\right)=0$, therefore $\bar{v}\left(\frac{a_{j}}{b^{n-j}}\right)=0$. We also have $\bar{w}\left(\frac{f}{b^{n}}\right)>w_{(a, \delta)}\left(\frac{f}{b^{n}}\right)=0$.

Consider the image of $\frac{f}{b^{n}}$ in $k_{\bar{w}}$. We have $\sum_{j=0}^{n} \frac{a_{j}}{b^{n-j}} t^{j}=0$ with the coefficient of $t^{n}$ equal to 1. This contradicts the fact that $t$ is transcendental over $k_{\bar{v}}$.

Conversely, suppose that $\bar{w}=w_{(a, \delta)}$ for $(a, \delta) \in \bar{K} \times \Gamma_{\bar{w}}$, with $\bar{w}(X-a)=\delta$. Then for all $b \in \bar{K}$ we have $X-b=X-a+a-b$, hence $\bar{w}(X-b)=\inf \{\delta, \bar{v}(a-b)\}$. Therefore $\bar{w}(X-b) \leq \bar{w}(X-a)$.

Lemma 3.5. Let $(a, \delta)$ be a pair of definition of $\bar{w}$. For each polynomial $f \in K[X]$ of degree $\operatorname{deg} f<\mathcal{D}(\bar{w})$, we have $w(f(X))=\bar{v}(f(a))$.

Proof. Take a polynomial $f \in K[X]$ of degree $r<\mathcal{D}(\bar{w})$. Let $b_{1} \ldots, b_{r}$ be the roots of $f$ (the $b_{i}$ need not be distinct).

For each $t, 1 \leq t \leq r$, we have $\bar{v}\left(a-b_{t}\right)<\delta$. Indeed, if there existed $t$ such that $\bar{v}\left(a-b_{t}\right) \geq \delta$, by Lemma $2.4\left(b_{t}, \delta\right)$ would be a pair of definition of $\bar{w}$, with $\left[K\left(b_{t}\right): K\right]<\mathcal{D}(\bar{w})$. This contradicts the definition of $\mathcal{D}(\bar{w})$.

Therefore, for each $t, 1 \leq t \leq r$, we have $\bar{w}\left(X-b_{t}\right)=\inf \left\{\delta, \bar{v}\left(a-b_{t}\right)\right\}=\bar{v}\left(a-b_{t}\right)$.

$$
\text { Now write } f(X)=c \prod_{t=1}^{r}\left(X-b_{t}\right)
$$


We have $w(f(X))=\bar{w}(f(X))=\bar{v}(c) \sum_{t=1}^{r} \bar{w}\left(X-b_{t}\right)=\bar{v}(c) \sum_{t=1}^{r} \bar{v}\left(a-b_{t}\right)=\bar{v}(f(a))$.

From Proposition 3.3 and Proposition 3.4 we deduce the following Corollary.

Corollary 3.6. The following conditions are equivalent:

(1) $w$ is valuation-transcendental extension of $v$ to $K(X)$

(2) for every algebraic closure $\bar{K}$ of $K$ and every extension $\bar{v}$ to $\bar{K}$ and a common extension of $w$ and $\bar{v}$ to $\bar{K}(X)$, there exists $(a, \delta) \in \bar{K} \times \Gamma_{\bar{w}}$ such that $\bar{w}=w_{(a, \delta)}$

(3) there exists an algebraic closure $\bar{K}$ of $K$, an extension $\bar{v}$ to $\bar{K}$ and a common extension of $w$ and $\bar{v}$ to $\bar{K}(X)$ such that $\bar{w}=w_{(a, \delta)}$ for a certain $(a, \delta) \in \bar{K} \times \Gamma_{\bar{w}}$.

\section{Key Polynomials}

In this section, we introduce the notion of key polynomials of a valuation and study some of their basic properties.

1. For each strictly positive integer $b$, we write $\partial_{b}:=\frac{\partial^{b}}{b ! \partial X^{b}}$, the $b$-th formal derivative with respect to $X$.

2. For each polynomial $f \in K[X]$, let $\epsilon(f):=\max _{b \in \mathbb{N}^{*}}\left\{\frac{w(f)-w\left(\partial_{b} P\right)}{b}\right\}$.

Definition 4.1. Let $Q$ be a monic polynomial in $K[X]$. We say that $Q$ is a key polynomial for $w$ if for each polynomial $f$ satisfying

$$
\epsilon(f) \geq \epsilon(Q)
$$

we have $\operatorname{deg}(f) \geq \operatorname{deg}(Q)$.

For a monic polynomial $Q \in K[X]$, every polynomial $f \in K[X]$ can be written in a unique way as

$$
f=\sum_{j=0}^{s} f_{j} Q^{j},
$$

with all the $f_{j} \in K[X]$ of degree strictly less than $\operatorname{deg}(Q)$. We call (4.1) the $Q$-expansion of $f$.

Definition 4.2. Let $g=\sum_{j=0}^{s} g_{j} Q^{j}$ be the $Q$-expansion of an element $g \in K[X] \backslash\{0\}$. We put

$$
w_{Q}(g):=\min _{\substack{0 \leq j \leq s \\ g_{j} \neq 0}} w\left(g_{j} Q^{j}\right)
$$

We adopt the convention that $w_{Q}(0)=\infty$. The mapping $w_{Q}: K[X] \rightarrow \Gamma_{w}$ is called the truncation of $w$ with respect to $Q$.

We have the following Proposition ([15], Proposition 2.4 (ii) and Proposition 2.6).

Proposition 4.3. If $Q$ is a key polynomial then $Q$ is irreducible and $w_{Q}$ is a valuation. 
For each polynomial $f(X) \in K[X]$, let $\delta(\bar{w}, f)=\max \{\bar{w}(X-a) / a \in \bar{K}, f(a)=0\}$.

Proposition 4.4. ([16], Proposition 3.1) Let $f(X) \in K[X]$ be a monic polynomial. We have $\delta(\bar{w}, f)=\epsilon(f)$.

The quantity $\delta(\bar{w}, f)$ depends only on $w$ and $f$, but not on $\bar{w}$, therefore we will denote it by $\delta(f)$.

Definition 4.5. Let $\Lambda$ be an ordered set. A set $\left\{Q_{i}\right\}_{i \in \Lambda}$ of key polynomials is said to be complete for $w$ if for every $f \in K[X]$ there exists $i \in \Lambda$ such that $w_{Q_{i}}(f)=w(f)$.

Remark 4.6. By [15], Theorem 1.1 and its proof, there is a complete set of key polynomials $\left\{Q_{i}\right\}_{i \in \Lambda}$ for the valuation $w$, having the following properties:

1. $\Lambda=\bigcup_{j \in I} \Lambda_{j}$, with $I=\{0, \ldots, N\}$ or $\mathbb{N}$, and for each $j$ we have $\Lambda_{j}=\{j\} \cup \vartheta_{j}$, where $\vartheta_{j}$ an ordered set without a maximal element, which may be empty.

2. There exists $a \in K$ such that $Q_{0}=X-a$.

3. For all $j \in I \backslash\{0\}$ we have $j-1<\vartheta_{j-1}<j$.

4. All the polynomials $Q_{i}$ with $i \in \Lambda_{j}$ have the same degree, and the polynomials $Q_{i}$ with $i \in \Lambda_{j}$ have degree strictly less than the polynomials $Q_{i^{\prime}}, i^{\prime} \in \Lambda_{j+1}$.

5. For each $i<i^{\prime} \in \Lambda$ we have $w\left(Q_{i}\right)<w\left(Q_{i}^{\prime}\right)$ and $\epsilon\left(Q_{i}\right)<\epsilon\left(Q_{i}^{\prime}\right)$.

For each $i \in \Lambda$, put $w_{i}=w_{Q_{i}}, \beta_{i}=w\left(Q_{i}\right)$ and $\epsilon_{i}=\epsilon\left(Q_{i}\right)$.

Even though the set of key polynomials $\left\{Q_{i}\right\}_{i \in \Lambda}$ is not unique, the cardinality of $I$ and the degrees $d_{j}$ of the key polynomials $Q_{j}$ for each $j \in I$ are uniquely determined by $w$. As well, the valuations $w_{j}$ for $j \in I$ are uniquely determined by $w$.

Remark 4.7. The above uniqueness statements together with conditions 1 and 3 above imply, in particular, that if $i \in \Lambda$ is not the maximal element then the set $\left\{Q_{j}\right\}_{\substack{j \in \Lambda \\ j \leq i}}$ of key polynomials is not complete (equivalently, $w_{i} \neq w$ ).

Notation. We will by denote by $d(w)$ the degree of $Q_{N}$ for the maximal element $N$ of $I$ if it exists. If $I=\mathbb{N}$, we put $d(w)=\infty$.

Remark 4.8. A direct consequence of the construction of [15] is that for all $i \in \Lambda_{j}$ the value group $\Gamma_{i}$ of $w_{i}$ is equal to $\Gamma_{v}+\beta_{0} \mathbb{Z}+\cdots+\beta_{j} \mathbb{Z}$.

Also by construction, $\Lambda$ has a maximal element if and only if the following two conditions hold:

1. the set $I=\{0, \ldots, N\}$ is finite and

2. $\Lambda_{n}=\{N\}$. 
Keep the above notation. Take an element $i \in \Lambda$.

Definition 4.9. We say that $Q_{i}$ is a limit key polynomial if the following two conditions hold:

1. $i \in I \backslash\{0\}$

2. $\vartheta_{i-1} \neq \emptyset$.

Proposition 4.10. There exists a complete set $\left\{Q_{i}\right\}_{i \in \Lambda}$ of key polynomials having the following additional property. For every $j \in I$ such that $\Lambda_{j+1} \neq \emptyset$ and $\vartheta_{j}=\emptyset$ (in other words, $Q_{j+1}$ is not a limit key polynomial), we can write $Q_{j+1}=q_{n} Q_{j}^{n}+\cdots+q_{t} Q_{j}^{t}+\cdots+q_{0}$, with $q_{n}=1$, $\operatorname{deg} q_{t}<d_{j}$ and $w\left(q_{t}\right)+t \beta_{j}=n \beta_{j}$ for each $t, 0 \leq t \leq n-1$.

For a proof of Proposition 4.10, one can imitate the proof of Theorem 9.4 [12] or Theorem 1.11 [20].

In the sequel, we will always choose a complete set $\left\{Q_{i}\right\}_{i \in \Lambda}$ of key polynomials satisfying the conclusion of Proposition 4.10 .

\section{Minimal Pairs and Key Polynomials}

Let $\left\{Q_{i}\right\}_{i \in \Lambda}$ be a complete set of key polynomials for $w$.

In this section, we study the relation between the properties of $\left\{Q_{i}\right\}_{i \in \Lambda}$, minimal pairs for a common extension $\bar{w}$, and the type of $w$ as residue-transcendental, value-transcendental or valuation-algebraic.

Proposition 5.1. Take an element $i \in \Lambda$. If $i$ is not the maximal element of $\Lambda$ then

$$
\Gamma_{i} \subset \Gamma_{v} \otimes_{\mathbb{Z}} \mathbb{Q}
$$

Proof. By Remark 4.8, it is sufficient to prove (5.1) for $i \in I$. Take an element $i \in I$. Suppose, inductively, that for each $i^{\prime} \in I, i^{\prime}<i$ we have $\beta_{i^{\prime}} \in \Gamma_{v} \otimes_{\mathbb{Z}} \mathbb{Q}$ (by Remark 4.8 this implies that $\beta_{j} \in \Gamma_{v} \otimes_{\mathbb{Z}} \mathbb{Q}$ for each $j \in \Lambda$ with $j<i$ ). Assume that $\beta_{i} \notin \Gamma_{v} \otimes_{\mathbb{Z}} \mathbb{Q}$, aiming for contradiction. For each element $g \in K[X]$, all the terms of its $Q_{i}$-expansion have different values, hence $w_{i}(g)=w(g)$. Since this holds for all $g \in K[X]$, we have $w_{i}=w$. By Remark 4.7, this is impossible since $i$ is not the maximal element of $\Lambda$.

Corollary 5.2. The valuation $w$ is a value-transcendental extension of $v$ if and only if $\Lambda$ has a maximal element $i_{0}$, that is $w=w_{i_{0}}, \beta_{i} \in \Gamma_{v} \otimes_{\mathbb{Z}} \mathbb{Q}$ for all $i<i_{0}$ and $\beta_{i_{0}} \notin \Gamma_{v} \otimes_{\mathbb{Z}} \mathbb{Q}$.

Proposition 5.3. If $\beta_{i} \in \Gamma_{v} \otimes_{\mathbb{Z}} \mathbb{Q}$ then $w_{i}$ is a residue-transcendental extension of $v$.

Proof. In what follows, we will adopt the convention that $\Gamma_{-1}:=\Gamma_{v}$. Let $j \in I$ be such that $i \in \Lambda_{j}$. Let $l=\min \left\{s / s \beta_{j} \in \Gamma_{j-1}\right\}$. There exists a polynomial $g(X)$ with $\operatorname{deg}(g)<\operatorname{deg}\left(Q_{i}\right)$ such that $w_{i}(g)=l \beta_{i}$.

Put $t=\left(\frac{Q_{i}^{l}}{g}\right)^{*}$ in $k_{w_{i}}$. Assume that $t$ is algebraic over $k_{v}$ and let

$$
t^{n}+a_{n-1} t^{n-1}+\cdots+a_{0}
$$


be an algebraic equation satisfied by $t$ over $k_{v}$. Choose representatives $a_{n-1}^{\prime}, \ldots, a_{0}^{\prime}$ in $K$ of the coefficients $a_{n-1}, \ldots, a_{0}$. Then $w_{i}\left(\left(\frac{Q_{i}^{l}}{g}\right)^{n}+a_{n-1}^{\prime}\left(\frac{Q_{i}^{l}}{g}\right)^{n-1}+\cdots+a_{0}^{\prime}\right)>0$. That is, $w_{i}\left(\left(Q_{i}^{l}\right)^{n}+a_{n-1}^{\prime} g\left(Q_{i}^{l}\right)^{n-1}+\cdots+a_{0} g^{n}\right)>w_{i}\left(g^{n}\right)=n l \beta_{i} \geq w_{i}\left(\left(Q_{i}^{l}\right)^{n}+a_{n-1}^{\prime} g\left(Q_{i}^{l}\right)^{n-1}+\cdots+a_{0} g^{n}\right)$, which is absurd.

Corollary 5.4. If $i$ is not the maximal element of $\Lambda$ then the valuation $w_{i}$ is residue-transcendental.

Proof. This is a direct consequence of Proposition 5.1 and Proposition 5.3

Proposition 5.5. If $M_{\bar{w}}$ has a maximal element $\delta$, then $\Lambda$ has a maximal element $i_{0}$ and there exists a root $a \in \mathcal{R}\left(Q_{i_{0}}\right)$ such that $(a, \delta)$ is a minimal pair of definition for $\bar{w}$.

Proof. Assume that $M_{\bar{w}}$ has a maximal element $\delta$. Let $(a, \delta)$ be a minimal pair of definition for $\bar{w}$ (it exists by Proposition 3.4. $)$.

Let $f(X)$ be the minimal polynomial of $a$ over $K$. The polynomial $f(X)$ is a key polynomial for $w$, since if $g(X)$ is such that $\epsilon(g) \geq \epsilon(f)$ then $\delta(g) \geq \delta(f)=\delta$ hence $\operatorname{deg}(g) \geq \operatorname{deg}(f)$, since $(a, \delta)$ is a minimal pair.

If there existed $i \in \Lambda$ such that $\epsilon\left(Q_{i}\right)>\epsilon(f)$, we would have $\delta\left(Q_{i}\right)>\delta(f)=\delta$ which is impossible by definition of $f$ and minimal pair of definition. Hence

$$
\epsilon\left(Q_{i}\right) \leq \epsilon(f) \quad \text { for all } i \in \Lambda .
$$

By definition of key polynomial, this implies that $\operatorname{deg}\left(Q_{i}\right) \leq \operatorname{deg}(f)$ for all $i \in \Lambda$.

Let $i \in \Lambda$ be such that $w_{i}(f)=w(f)$. By [15] Proposition 2.10 (ii) we must have $\epsilon\left(Q_{i}\right) \geq \epsilon(f)$, hence $\epsilon\left(Q_{i}\right)=\epsilon(f)$ in view of (5.2). We conclude that $\delta\left(Q_{i}\right)=\delta$.

Choose $a^{\prime} \in \mathcal{R}\left(Q_{i}\right)$, such that $\bar{w}\left(X-a^{\prime}\right)=\delta$. By Lemma 2.4 $\left(a^{\prime}, \delta\right)$ is a pair of definition for $\bar{w}$, and since $\mathcal{D}(\bar{w})=\left[K\left(a^{\prime}\right): K\right]$, we have that $\left(a^{\prime}, \delta\right)$ is a minimal pair of definition for $\bar{w}$.

Now $i$ must be the greatest element of $\Lambda$ since otherwise, if there exists $i^{\prime}>i$, by Remark 4.63 we have $e\left(Q_{i^{\prime}}\right)>e\left(Q_{i}\right)$ and $\delta\left(Q_{i^{\prime}}\right)>\delta\left(Q_{i}\right)=\delta$, which is impossible.

Theorem 5.6. The valuation $w$ is a valuation-transcendental extension of $v$ if and only if $\Lambda$ has a maximal element.

Moreover, in this case, if $\left\{Q_{i}\right\}_{i \in \Lambda}$ is a complete set of key polynomials for $w$ and $i_{0}$ the maximal element of $\Lambda$, then for every common extension $\bar{w}$ of $\bar{v}$ and $w$ to $\bar{K}(X), \mathcal{D}(\bar{w})=d(w)$ and there exists $a \in \mathcal{R}\left(Q_{i_{0}}\right)$ such that $w_{\left(a, \epsilon_{i_{0}}\right)}=\bar{w}$.

Proof. Let $\bar{w}$ be a common extension of $\bar{v}$ and $w$ to $\bar{K}(X)$. By Proposition $3.3 w$ is valuationtranscendental if and only if $M_{\bar{w}}$ has a maximal element.

Now if $w$ is valuation-transcendental, by Proposition 5.5, $\Lambda$ has a maximal element.

Conversely, if $\Lambda$ has a maximal element, by Corollary 5.2 and Proposition 5.3, $w$ is valuation-transcendental. 
The last statement of the Theorem is a direct consequence of Proposition 5.5 .

Corollary 5.7. If $n$ is the number of distinct roots of the final key polynomial in $\left\{Q_{i}\right\}_{i \in \Lambda}$, then there exist at most $n$ common extensions of $w$ and $\bar{v}$ to $\bar{K}(X)$.

Example 5.8. The pair $\left(0, \beta_{0}\right)=(0, w(X-a))=\left(0, \epsilon_{0}\right)$ is a minimal pair for the valuation $w_{0}$ (see Remark 4.6 2 and the notation that follows it).

\section{Common Extensions}

Let $\left\{Q_{i}\right\}_{i \in \Lambda}$ be a complete set of key polynomials for $w$.

By Theorem 5.6, if $w$ is a valuation-transcendental extension of $v$ then $w=w_{i_{0}}$, where $i_{0}$ is the maximal element of $\Lambda$, and if $\bar{w}$ is a common extension of $w$ and $\bar{v}$ to $\bar{K}(X)$, then there exists $a \in \mathcal{R}\left(Q_{i_{0}}\right)$, such that $\bar{w}:=w_{(a, \delta)}$, where $\delta=\max \left(M_{\bar{w}}\right)=\epsilon\left(Q_{i_{0}}\right)$.

In this section, we investigate which roots $a \in \mathcal{R}\left(Q_{i_{0}}\right)$ are such that $w_{(a, \delta)}$ is a common extension of $w$ and $\bar{v}$. By definition of $w_{(a, \delta)}$, it is an extension of $\bar{v}$, hence the question is if the restriction of $w_{(a, \delta)}$ to $K(X)$ is equal to $w$.

Note that for each $i \in \Lambda$, since the valuation $w_{i}$ is a valuation-transcendental extension of $v$, we have that every common extension of $\bar{v}$ and $w_{i}$ to $\bar{K}(X)$ has the form $w_{\left(b, \epsilon_{i}\right)}$, with $b \in \mathcal{R}\left(Q_{i}\right)$ (Proposition 3.4).

Assume that we know a minimal pair of definition $(a, \delta)$ for a common extension $\bar{w}$ of $\bar{v}$ and $w$ to $\bar{K}(X)$. The following Lemma gives a criterion to characterize the other minimal pairs of definition for common extensions of $\bar{v}$ and $w$ to $\bar{K}(X)$.

Lemma 6.1. Let $\bar{w}$ be a common extension of $\bar{v}$ and $w$ to $\bar{K}(X)$ and let $(a, \delta)$ be a minimal pair for $\bar{w}$. Let $f$ be the minimal polynomial of a over $K$ and let $b \in \mathcal{R}(f)$. Then $w_{(b, \delta)}$ is a common extension of $\bar{v}$ and $w$ to $\bar{K}(X)$ if and only if for every $g \in K[X]$ with $\operatorname{deg}(g)<d(w)$, we have $w(g(X))=\bar{v}(g(b))$.

Proof. Suppose first that $w_{(b, \delta)}$ is a common extension of $\bar{v}$ and $w$. By Theorem 5.6 we have $\mathcal{D}\left(w_{(b, \delta)}\right)=d(w)$. By Lemma 3.5, for every $g \in K[X]$ with $\operatorname{deg}(g)<d(w)$ we have $\bar{v}(g(b))=w(g(X))$.

Conversely, suppose that for every $g \in K[X]$ with $\operatorname{deg}(g)<d(w)$ we have

$$
\bar{v}(g(b))=w(g(X)) .
$$

We claim that for every $g \in K[X]$ we have $\bar{v}(g(a)))=\bar{v}(g(b))$.

By Lemma 3.5 we have $\bar{v}(g(a)))=w(g(X))$ for every $g \in K[X]$ with $\operatorname{deg}(g)<d(w)$. Hence for every $g \in K[X]$ with $\operatorname{deg}(g)<d(w)$, we have $\bar{v}(g(a))=\bar{v}(g(b))$. We still need to prove that for every $g \in K[X]$ with $\operatorname{deg}(g) \geq d(w)$ we have $\bar{v}(g(a)))=\bar{v}(g(b))$.

Consider $g \in K[X]$ with $\operatorname{deg} g \geq d(w)$. Since $(a, \delta)$ is a minimal pair, $d(w)=\operatorname{deg}(f)$. Let $g(X)=q(X) f(X)+r(X)$ be the Euclidean division of $g(X)$ by $f(X)$, with $\operatorname{deg} r(X)<d(w)$. 
We have $g(a)=r(a)$ and $g(b)=r(b)$. Since we already know that $\bar{v}(r(a))=\bar{v}(r(b))$, the claim is proved.

We want to prove that $w_{(b, \delta)}$ is equal to $w$ on $K(X)$. For this it is sufficient to prove that it is equal to $w$ on $K[X]$, therefore it is sufficient to prove that for every $g(X) \in K[X]$ we have $w_{(a, \delta)}(g(X))=w_{(b, \delta)}(g(X))$.

Take $g(X) \in K[X]$ and write the Taylor expansions of $g(X)$ :

$$
\begin{aligned}
& g(X)=g_{n}(a)(X-a)^{n}+\cdots+g_{0}(a), \\
& g(X)=g_{n}(b)(X-b)^{n}+\cdots+g_{0}(b),
\end{aligned}
$$

where for each $t, 0 \leq t \leq n$, we have that $g_{t}(X)=\partial_{t} g(X)$ is a polynomial in $K[X]$, hence, by the above discussion, $\bar{v}\left(g_{t}(a)\right)=\bar{v}\left(g_{t}(b)\right)$.

Now by definition of $w_{(a, \delta)}$ and of $w_{(b, \delta)}$ we have

$$
\begin{aligned}
w_{(a, \delta)}(g(X)) & =\inf _{0 \leq t \leq n}\left\{\bar{v}\left(g_{t}(a)\right)+t \delta\right\} \\
& =\inf _{0 \leq t \leq n}\left\{\bar{v}\left(g_{t}(b)\right)+t \delta\right\} \\
& =w_{(b, \delta)}(g(X)) .
\end{aligned}
$$

Lemma 6.2. Let $i \in \Lambda$ and suppose that for every $b \in \mathcal{R}\left(Q_{i}\right), \bar{w}_{i}:=w_{\left(b, \epsilon_{i}\right)}$ is an extension of $w_{i}$. Let $\alpha \in \bar{K}$. If for every root $b \in \mathcal{R}\left(Q_{i}\right)$ we have $\bar{v}(\alpha-b)<\epsilon_{i}$ then $\bar{v}\left(Q_{i}(\alpha)\right)<w_{i}\left(Q_{i}\right)=\beta_{i}$.

Proof. Choose $b \in \mathcal{R}\left(Q_{i}\right)$ such that $\bar{v}(\alpha-b) \geq \bar{v}(\alpha-c)$ for every $c \in \mathcal{R}\left(Q_{i}\right)$. Let $\bar{w}_{i}:=w_{\left(b, \epsilon_{i}\right)}$. We will prove that

$$
\bar{w}_{i}(X-c) \geq \bar{v}(\alpha-c) \text { for every } c \in \mathcal{R}\left(Q_{i}\right)
$$

For every $c \in \mathcal{R}\left(Q_{i}\right)$, we have, $\bar{w}_{i}(X-c)=\inf \left\{\epsilon_{i}, \bar{v}(b-c)\right\}$.

Now, $\epsilon_{i}>\bar{v}(\alpha-c)$ by assumption and $\bar{v}(b-c) \geq \inf \{\bar{v}(b-\alpha)$ and $\bar{v}(\alpha-c)\} \geq \bar{v}(\alpha-c)$, where the last inequality follows from the definition of $b$.

We have $Q_{i}(X)=\prod_{l}\left(X-c_{l}\right)$, where the $c_{l}$ are the roots of $Q_{i}$, which need not be distinct. Now the result follows from the facts that $w_{i}\left(Q_{i}(X)\right)=\bar{w}_{i}\left(Q_{i}(X)\right), \bar{w}_{i}(X-b)=\epsilon_{i}>\bar{v}\left(\alpha-c_{l}\right)$ and (6.1).

Lemma 6.3. Let $j \in I$ be such that $\vartheta_{j}=\emptyset$ and for every $b \in \mathcal{R}\left(Q_{j}\right)$ the valuation $\bar{w}_{b j}:=w_{\left(b, \epsilon_{j}\right)}$ is an extension of $w_{j}$. Assume that $\Lambda_{j+1} \neq \emptyset$ and let $a \in \mathcal{R}\left(Q_{j+1}\right)$. If there exists $b \in \mathcal{R}\left(Q_{j}\right)$ such that $\bar{v}(a-b) \geq \epsilon_{j}$ then $\beta_{j}=w_{j}\left(Q_{j}\right)=\bar{v}\left(Q_{j}(a)\right)$. 
Proof. Choose $b \in \mathcal{R}\left(Q_{j}\right)$ such that $\bar{v}(a-b) \geq \epsilon_{j}$, and let $\bar{w}_{b j}:=w_{\left(b, \epsilon_{j}\right)}$. By Lemma 2.4, $\left(a, \epsilon_{j}\right)$ is also a pair of definition for $\bar{w}_{b j}$.

By Proposition 4.10 and the comment that follows it, we can write

$$
Q_{j+1}=q_{n} Q_{j}^{n}+\cdots+q_{t} Q_{j}^{t}+\cdots+q_{0}, \quad \text { with } q_{n}=1, \operatorname{deg} q_{t}<d_{j}
$$

and

$$
w\left(q_{t}\right)+t \beta_{j}=n \beta_{j} \quad \text { for } t \in\{0, \ldots, n\} .
$$

By (6.2), given integers $t_{1}, t_{2}$ with $0 \leq t_{1}<t_{2}$, we have $w\left(q_{t_{1}}\right)+t_{1} \beta_{j}=w\left(q_{t_{2}}\right)+t_{2} \beta_{j}$, so $w\left(Q_{j}(X)\right)=\beta_{j}=\frac{w\left(q_{t_{2}}(X)\right)-w\left(q_{t_{1}}(X)\right)}{t_{2}-t_{1}}$. Combining this with Lemma 3.5, we obtain

$$
w\left(Q_{j}(X)\right)=\frac{\bar{v}\left(q_{0}(a)\right)}{n} .
$$

Now $0=Q_{j+1}(a)=Q_{j}^{n}(a)+\cdots+q_{t}(a) Q_{j}^{t}(a)+\cdots+q_{0}(a)$. Therefore, there exist $t_{1}, t_{2}$, $0 \leq t_{1}<t_{2} \leq n$ such that $\bar{v}\left(q_{t_{1}}(a) Q_{j}^{t_{1}}(a)\right)=\bar{v}\left(q_{t_{2}}(a) Q_{j}^{t_{2}}(a)\right)$.

Thus we have $\bar{v}\left(Q_{j}(a)\right)=\frac{\bar{v}\left(q_{t_{2}}(a)\right)-\bar{v}\left(q_{t_{1}}(a)\right)}{t_{2}-t_{1}}=w\left(Q_{j}(X)\right)$.

Lemma 6.4. Let $j \in I$ be such that $\vartheta_{j}=\emptyset$ and for every $b \in \mathcal{R}\left(Q_{j}\right)$ the valuation $\bar{w}_{b j}:=w_{\left(b, \epsilon_{j}\right)}$ is an extension of $w_{j}$. Assume that $\Lambda_{j+1} \neq \emptyset$. Then for every root $a \in \mathcal{R}\left(Q_{j+1}\right)$ there exists $b \in \mathcal{R}\left(Q_{j}\right)$ such that $\bar{v}(a-b) \geq \epsilon_{j}$.

Proof. By Proposition 4.10 and the comment that follows it, we can write

$$
Q_{j+1}=q_{n} Q_{j}^{n}+\cdots+q_{t} Q_{j}^{t}+\cdots+q_{0}, \quad \text { where } q_{n}=1, \operatorname{deg} q_{t}<d_{j} \text { and (6.2) is satisfied. }
$$

Let $b_{1}, \ldots, b_{t}$ be the roots of $Q_{j}$ and $a_{1}, \ldots, a_{s}$ the roots of $Q_{j+1}$ (not necessarily distinct). We have $s=n \cdot t$ and the resultant of $Q_{j}$ and $Q_{j+1}$ is given by

$$
\prod_{\ell=1}^{t} Q_{j+1}\left(b_{\ell}\right)=(-1)^{n t^{2}} \prod_{k=1}^{s} Q_{j}\left(a_{k}\right)
$$

For each $\ell, 1 \leq \ell \leq t$, we have $\bar{v}\left(Q_{j+1}\left(b_{\ell}\right)\right)=\bar{v}\left(q_{0}\left(b_{\ell}\right)\right)=w_{j}\left(q_{0}(X)\right)=n \beta_{j}$, where the second equality is obtained from Lemma 3.5. We obtain

$$
\bar{v}\left(\prod_{k=1}^{s} Q_{j}\left(a_{k}\right)\right)=\bar{v}\left(\prod_{\ell=1}^{t} Q_{j+1}\left(b_{\ell}\right)\right)=\operatorname{tn} \beta_{j}=s \beta_{j} .
$$

Hence

$$
\sum_{k=1}^{s} \bar{v}\left(Q_{j}\left(a_{k}\right)\right)=s \beta_{j} .
$$

By Lemmas 6.2 and 6.3 we have, for each $k, 1 \leq k \leq s, \bar{v}\left(Q_{j}\left(a_{k}\right)\right) \leq \beta_{j}$ (where we apply Lemma 6.2 in the case when for every root $b \in \mathcal{R}\left(Q_{i}\right)$ we have $\bar{v}\left(a_{k}-b\right)<\epsilon_{i}$ and Lemma 6.3 in the case when there exists $b \in \mathcal{R}\left(Q_{j}\right)$ such that $\left.\bar{v}\left(a_{k}-b\right) \geq \epsilon_{j}\right)$. Hence, in view of (6.5) we must have the equality $\bar{v}\left(Q_{j}\left(a_{k}\right)\right)=\beta_{j}$, for each $k, 1 \leq k \leq s$.

Therefore, by Lemma 6.2, for each $k, 1 \leq k \leq s$, there exists $\ell, 1 \leq \ell \leq t$, such that $\bar{v}\left(a_{k}-b_{\ell}\right) \geq \epsilon_{j}$. 
Proposition 6.5. Let $j \in I$ be such that $\vartheta_{j}=\emptyset$ and consider $b \in \mathcal{R}\left(Q_{j}\right)$ such that $\bar{w}_{b j}:=w_{\left(b, \epsilon_{j}\right)}$ is an extension of $w_{j}$. Assume that $\Lambda_{j+1} \neq \emptyset$, and let $a \in \mathcal{R}\left(Q_{j+1}\right)$ be such that $\bar{v}(a-b) \geq \epsilon_{j}$. Then $w_{\left(a, \epsilon_{j+1}\right)}$ is an extension of $w_{j+1}$.

Proof. Let $\bar{w}_{j+1}$ be a common extension of $\bar{v}$ and $w_{j+1}$ to $\bar{K}(X)$. By Theorem 5.6, there exists a root $a_{1}$ of $Q_{j+1}$ such that $\bar{w}_{j+1}=w_{\left(a_{1}, \delta\right)}$. If $a=a_{1}$, we are done, there is nothing to prove. Otherwise, assume that $a \neq a_{1}$.

In view of Lemma 6.1, it is sufficient to prove that for all $g \in K[X]$ with $\operatorname{deg} g<d_{j+1}$ we have $\bar{v}(g(a))=w(g(X))$.

By Lemma 2.4, $\left(a, \epsilon_{j}\right)$ is also a pair of definition for $\bar{w}_{b j}$. Therefore, by Lemma 3.5,

for all $g \in K[X]$ with $\operatorname{deg} g<d_{j}$ we have $\bar{v}(g(a))=w_{j}(g(X))=w(g(X))$.

By Lemma 6.3, we have $\bar{v}\left(Q_{j}(a)\right)=\beta_{j}$.

We will first prove, by contradiction, that for all $g \in K[X]$ with $\operatorname{deg} g<d_{j+1}$, we have $\bar{v}(g(a)) \geq w(g(X))$.

Assume that there exists $g \in K[X], \operatorname{deg} g<d_{j+1}$, such that $\bar{v}(g(a))<w(g(X))$. Choose $g$ to be of minimal degree subject to this inequality. By (6.6) we must have

$$
\operatorname{deg} g \geq d_{j}
$$

Let $g(X)=q(X) Q_{j}(X)+r(X)$ be the Euclidean division of $g(X)$ by $Q_{j}(X)$, with

$$
\operatorname{deg} r<d_{j}
$$

Note that

$$
\operatorname{deg} q<\operatorname{deg} g
$$

We have

$$
\begin{aligned}
\bar{v}(g(a)) & \geq \inf \left\{\bar{v}(q(a))+\bar{v}\left(Q_{j}(a)\right), \bar{v}(r(a))\right\} \\
& \geq \inf \left\{w_{j}(q(X))+w_{j}\left(Q_{j}(X)\right), w_{j}(r(X))\right\} \\
& =w_{j}(g(X)) \\
& =w(g(X)),
\end{aligned}
$$

where the second inequality follows from (6.6), (6.8), the assumed minimality of deg $g$ and the fact that $\left(a, \epsilon_{j}\right)$ is a pair of definition for $\bar{w}_{b j}$ which extends $w_{j}$. The above inequality contradicts our assumption.

Again, aiming for contradiction, we will assume that there exists

$$
g \in K[X], \quad d_{j} \leq \operatorname{deg} g<d_{j+1}
$$

such that $\bar{v}(g(a))>w(g(X))$. Assume that $g$ is chosen of minimal degree subject to this inequality.

Let $Q_{j+1}=Q g+R$ be the Euclidean division of $Q_{j+1}(X)$ by $g(X)$, with $\operatorname{deg} R<\operatorname{deg} g$. 
We have $w\left(Q_{j+1}\right)>w(Q g)=w(R)$, since $w_{j}(Q g)=w(Q g)$ and $w_{j}(R)=w(R)$ but $w\left(Q_{j+1}\right)>w_{j}\left(Q_{j+1}\right)$.

Moreover, we have $0=Q_{j+1}(a)=Q(a) g(a)+R(a)$. Therefore, we must have

$$
\bar{v}(R(a))=\bar{v}(Q(a) g(a)) .
$$

But $\bar{v}(R(a))=w(R(X))$ by the assumed minimality of the degree of $g$. Hence we must have $w(Q(X) g(X))=\bar{v}(Q(a) g(a))$, but $w(Q(X)) \leq \bar{v}(Q(a))$ and $w(g(X))<\bar{v}(g(a))$ which is impossible.

As a direct consequence of Lemma 6.4 and Proposition 6.5 we have the following Theorem:

Theorem 6.6. Let $j \in I$ be such that $\vartheta_{j}=\emptyset$, and for every $b \in \mathcal{R}\left(Q_{j}\right), \bar{w}_{b j}:=w_{\left(b, \epsilon_{j}\right)}$ is an extension of $w_{j}$. Assume that $\Lambda_{j+1} \neq \emptyset$. Then for every root $a \in \mathcal{R}\left(Q_{j+1}\right), w_{\left(a, \epsilon_{j+1}\right)}$ is an extension of $w_{j+1}$.

Corollary 6.7. Let $j_{0} \in I$ be such that $w=w_{j_{0}}$ and assume that for every $j \in I$ we have $\vartheta_{j}=\emptyset$. Then for every root $a \in \mathcal{R}\left(Q_{j_{0}}\right)$ the valuation $w_{\left(a, \epsilon_{j_{0}}\right)}$ is an extension of $w$.

Proof. We use induction on $j \leq j_{0}$. The base of the induction is nothing but Example 5.8. The induction step is given by Theorem 6.6 .

\section{References}

[1] V. Alexandru, A. Zaharescu, A theorem of characterization of residual transcendental extensions of a valuation, J. Math. Kyoto Univ. 28 (1988), 579-592.

[2] V. Alexandru, N. Popescu, A. Zaharescu, Minimal pairs of definition of a residual transcendental extension of a valuation, J. Math. Kyoto Univ. 30 (1990), 207-225.

[3] M. Alberich-Carraminana, A. F. Boix, J. Fernández, J. Guàrdia, E. Nart, J. Roé Invariants of limit key polynomials., arXiv:2005.04406vi 1 [math.AG].

[4] S. D. Cutkosky, H. Mourtada, B. Teissier On the construction of valuations and generating sequences on hypersurface singularities., arXiv:1904.10702v1 [math.AG].

[5] J. Decaup, W. Mahboub, M. Spivakovsky, Abstract key polynomials and comparison theorems with the key polynomials of MacLane-Vaquié, Illinois J. Math. 62, Number 1-4 (2018), 253-270.

[6] I. M. Gelfand, M. M. Kapranov, A. V. Zelevinsky, (1994), Discriminants, resultants, and multidimensional determinants, Boston: Birkhäuser, ISBN 978-0-8176-3660-9

[7] F. J. Herrera Govantes, M. A. Olalla Acosta, M. Spivakovsky, Valuations in algebraic field extensions, Journal of Algebra, Volume 312, Issue 2 (2007), pages 1033-1074.

[8] F. J. Herrera Govantes, W. Mahboub, M. A. Olalla Acosta, M. Spivakovsky Key polynomials for simple extensions of valued fields., arXiv:1406.0657v3 [math.AG].

[9] O. Kashcheyeva, Constructing examples of semigroups of valuations, J. Pure Appl. Algebra 220 (2016), 3826-3860 
[10] F.-V. Kuhlmann, Value groups, residue fields and bad places of rational function fields, Trans. Amer. Math. Soc. 356 (2004), 4559-4600.

[11] S. MacLane, A construction for prime ideals as absolute values of an algebraic field, Duke Math. J., vol 2 (1936), 492-510.

[12] S. MacLane A construction for absolute values in polynomial rings, Transactions of the AMS, vol 40 (1936), 363-395.

[13] W. Mahboub Key Polynomials, Journal of Pure and Applied Algebra, 217(6) (2013), pages 989-1006.

[14] E. Nart, Key polynomials over valued fields, Publ. Mat. 64 (2020), 3-42.

[15] J. Novacoski and M. Spivakovsky Key polynomials and pseudo-convergent sequences, Journal of Algebra 495 (2018), 199-219.

[16] J. Novacoski Key polynomials and minimal pairs, Journal of Algebra 523 (2019), 1-14.

[17] J.-C. San Saturnino, Defect of an extension, key polynomials and local uniformization, Journal of Algebra 481 (2017), 91-119.

[18] J.-C. San Saturnino, Ramification theory and key polynomials., arXiv:1602.08284v1 [math.AG].

[19] M. Vaquié, Famille admise associée à une valuation de $K[x]$. [Admissible family associated with a valuation of $K[x]$ ], Singularités Franco-Japonaises, 391-428, Sémin. Congr., 10, Soc. Math. France, Paris, 2005.

[20] M. Vaquié, Extension d'une valuation. [Extension of a valuation], Trans. Amer. Math. Soc. 359 (2007), no. 7, 3439-3481. (electronic)

[21] M. Vaquié, Famille admissible de valuations et défaut d'une extension. [Admissible family of valuations and defect of an extension], J. Algebra 311 (2007), no. 2, 859-876.

[22] M. Vaquié, Extensions de valuation et polygone de Newton. [Valuation extensions and Newton polygon] Ann. Inst. Fourier (Grenoble) 58 (2008), no. 7, 2503-2541.

[23] M. Vaquié, Valuation augmentée et paire minimale, arXiv:2005.03298v1 [math.CA]. 